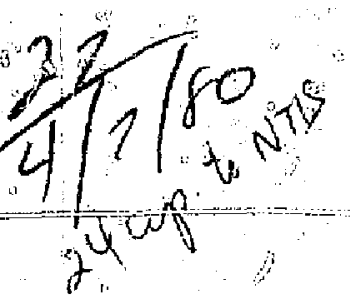

\title{
Modeling of Beam Focuging and Kink Instability for Colliding Relacivistic Electron and Positron Beams
}

William M. Fawley and Edward P. Lee

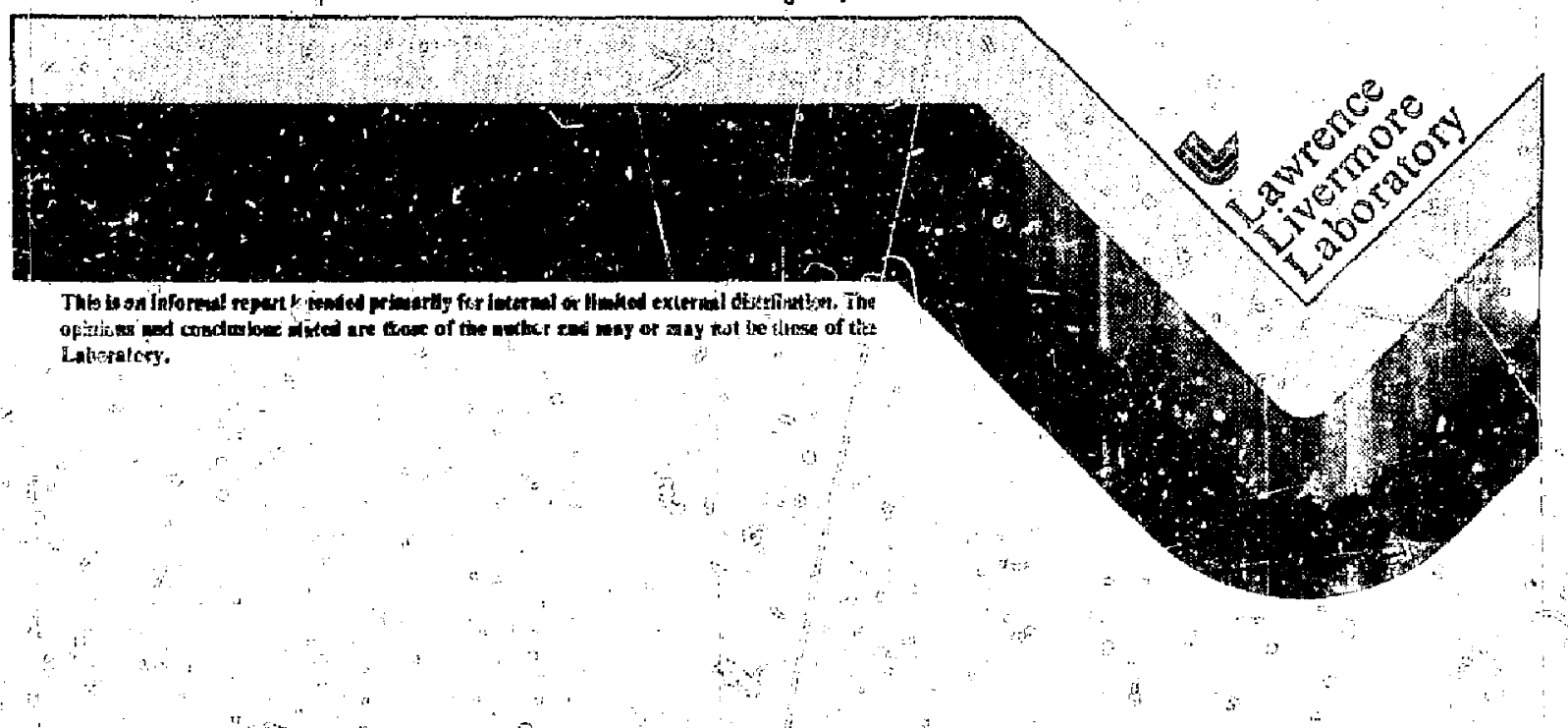

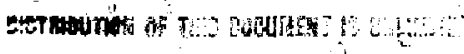




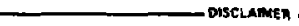

Mndeling of Beam Focusing and Kink Instarility for Colliding Relativistic Electron and Positron Beams

William M. Fawley and Edward P. Lee

Abstract.

A simulation code has been developed and exercised t.J study the time evolution and resultant liminosity of intersecting pulses of electrons and positrons. Under the extreme conditions of high current and small radius recentiy proposed for the SLAC facility. the pulses mutually pinch to a smaller mean radius than that achieved hy free flight alone. The effectiva Tuminosity is enhanced by a factor of $\approx 3$ in the hest. case. corresponding to zero initial emittance and pulse iength equa? $1 / M$ the mean hetatmon wavelength. Gatissian profiles in the longitudiral and transverse coordinates are preferred over flat profiles in order to minimize disruptive oscillations in radius which reduce luminosity.

A second potent, al source of disruption is the kink instability. This is always present to some degree furing the interaction of opposed pulses. However, the maximum growit's rate is only one half the angular betatron frequency and therefore mode growth is limited to a very low level if $\dagger \Rightarrow$ pulses are short compared with a betatron wavelength.

This work is jointly performed under the auspices of the U.S. Department of Energy by LLL, Contract No. W-7405-Eng-48 and DARPA (DOD), ARPA Order No. 3718. Monitored by NSHC under contract \#N60927-80-WR-WD188, Amendment $\$ 12$.

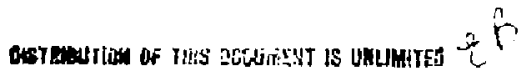


I. Introduction

SLAC has proposed to study the physics of intermediate vector boson particle $\left(7^{\circ}\right)$ creation by using colliding heams of $50 \mathrm{GeV}$ electrons and positrons. An interesting aspect of these proposed colliding beam experiments is that each beam will tend to focus the other. In coordination with SLAC, we decided to model these focussing effects and also to examine the possibility of a kink-type instability. The results presented here should re considered as complimentary to those of $R$. Hollebeek (SLAC) who has also studied beam focusing in this proposed experiment. \$II discusses the simulation code, \$ 111 contains the code results concerning luminosity. and $\xi$ IV is an analysis of the kink instability in the proposed experiments. 


\section{Simulation Code}

The uncertainties in mndelling the beam interaction are largely numerical; therefore to supplement R. Hollebeek's results (in which a Lagrangian grid approach was utilized), we decided to use a cloud in cell (CIC) simulation code. The code is axially symetric with particle coordinates being specified by $(r, z)$ and angular momentur only. The large relativistic Lorentz factors $\left(\sim 10^{5}\right)$ of the beam particles allowed us to use the paraxial approximation and set $v_{z}$ constant and equal to the speed of light $c$.

In the limit $Y \gg 1$, the self-anti pinch forces of each beam are of order $\mathrm{Y}^{-2}$ and may be safely neglacted relative to the electric and magnetic forces of the other beam. Thus, the equations of motion are

$$
\begin{aligned}
& m r \frac{d \underline{V}_{ \pm}}{d \bar{t}}= \pm e(\underline{\underline{E}} \pm \hat{e} z \times \underline{B}) \\
& =\frac{-4 e \hat{r}}{r} \int_{0}^{r}\left|\rho_{\mp}\left(r,,^{\prime} z\right)\right| 2 \pi r^{\prime} d r^{\prime} \equiv \frac{-\Delta e \hat{r}}{r}\left|Q_{\mp}(r . z)\right| .
\end{aligned}
$$

Here $P_{f}\left(r^{\prime} .7\right)$ is the charge density of the opposite beam and $e$ and $m$ are the electron charge and mass respectively. In deriving eq. (1), we have neglerted the $E_{z}$ and $B_{r}$ field components, which are of order $\gamma^{-1}(\ell / \lambda)$ where $\lambda$ is the scale height for changes in the $z$ direction and $\ell$ is the beam length. This approximation is equivalent to assuming the electromagnetic fields of each particle are totally contained in a plane transverse to $z$.

Employing the conserved angular inomentum $\left(L=\gamma m r v_{\theta}\right)$, eq. (1) nav be rewritten for the radial motion alone: 


$$
m \gamma \frac{d v_{r_{ \pm}}}{d t}=\frac{-4 e \mid Q_{\mp}}{r} \mid+\frac{L^{2}}{r m r^{3}}
$$

The other necessary equations are

$$
\frac{d r}{d t}=v_{r} \quad \text { and } \quad \frac{d z_{ \pm}}{d t}= \pm c
$$

The pancake-jike transverse fields associated each particle permit one to divine up each beam into a set of discs moving in the 2 direction, each of which is then gridder in the $r$ direction. Particles mav cross grid boundaries in $r$ but not in $z$ due to the paraxial and high $\gamma$ approximations. Although there is complete symmetry between electrons and positrons, it is convenjent to follow individual positron discs through the entire electron beam. After one such disc has completely traversed the electron heam, the next positron disc is loaded into memory and sent on its way. This method minimizes machine memory requirements because only one positron disc is present at any one time (though all the coordinates of the electron beam particies must be cont inuously kept).

A standard leap-frog scheme is used (see Fig. 1) in which particle positions are advanced on the half-step and fields and accelerations are calculated on the whole step. Unlike normal plasma simulations in which the time step $\Delta t$ is determined hy the size of the plasma frequency $\omega_{p}$, here the relevant frequency is the betatron frequency $\omega_{\beta}$. where

$$
\omega_{\beta}^{2}(r, z)=\frac{4 e Q(r, z)}{Y m r^{2}}
$$


A factor of 4 rather than 2 appears because both charge and current contrihute to the pinch force. For a constant beam charge density en,

$$
\begin{aligned}
\omega_{B}{ }^{2} & =\frac{\Delta \pi n e^{2}}{Y m} \\
& =\frac{\omega_{p}^{2}}{\gamma} .
\end{aligned}
$$

where $\omega_{p}$ is the plasma frequency and $\frac{\omega_{p}}{\gamma / 12}$ is the effective relativist ic transverse plasma frequency. In order to prevent numerical instability and to accurately follow particle oscillations, one wants $\omega_{\beta} \Delta t \leq 0$ (1). Since $\Delta t=\frac{\Delta z}{2 C}$ where $\Delta z$ is the thickness of an individual disc (the factor of 2 is due to the fact that the beams are moving through each other at a relative velocity of $2 c$ in the laboratory frame), one has

$$
\omega_{B}{ }^{2} \Delta t^{2} \approx \frac{\pi n e^{2}(\Delta z)^{2}}{\gamma m c^{2}} \approx \frac{\pi N_{ \pm} r_{e} \sigma_{z}}{\gamma \sigma_{x} \sigma_{y}(N G)^{2}}=\frac{\pi D}{2(N G)^{2}},
$$

where $r_{p}$ is the classical radius of the electron, $\left(\sigma_{x} \cdot \sigma_{y} \cdot \sigma_{z}\right)$ are the Gaussian widths of the beams in the 3 dimensions, NG is the number of discs, and $D$ is the "Disruption factor" as defined by $R$. Hollebeek. In general, $0 \leq 10$ and $N G \geq 15$ giving $\omega_{\beta} \Delta t \leq 0,2$.

Equation (2) has an absolute singularity at $r=0$ which makes the effective betatron frequency $\rightarrow \infty$ for finite angular momentum. To prevent this artificial instability from affecting the results the following "fix" was applied. Whenever a particle approached to within 3 grid cells of the central axis, Cartesian $x-y$ coordinates were used in the particle "mover" and "acceleration" subroutine. After moving the particle. a new $r$ and $v_{r}$ were 
caiculated while keeping the anguiar morientum constant.

Resolution requirements determine the radial gridding. Generally. 15 to 18 cells per disc are sufficient to ensure accurate determination of the fields and to allow space for heam expansion. if any, due to finite emittance. We use a cloud in cell approach (see reference (1)) to weight particles to the grid. Essentially, each particle is thought of as a cloud whose radial dimension is that of the grid spacing $\Delta r$ and whose charge density is assigned to the 2 nearest grid points. However, since the electramagnetic fields at a given radial point are determined by the charge interior to that point, one must add $0.5 \Delta r$ to each particle coordinate before cinarge assignation. This ensures that two particles with the same coordinate only "see" half of each other's charge. A similar weighting (without the addition of $\Delta r / 2)$ of fields to the particles is used. Thus

$$
E(r)=E^{n+1}\left(n+1-\frac{r}{\Delta r}\right)+E^{n}\left(\frac{r}{\Delta r}-n\right)
$$

for $n<r / \Delta r<n+1$ where $\varepsilon^{n}$ is the electric field at grid point $n$. For Cartesian grids. this weighting algorithm conseryes momentum; it is unclear as to whether this wiil be true for radial grids also.

Initially, we assigned both beams either flat or a gaussian profile in $r$ and z. An individual particle's angular momenta and $v_{r}$ were assigned randomly for a given beam emittance. Approximately 400 particles per disc were used to obtain statistical accuracy of $10 \%$ or better.

\section{Simulation Results}

Two sets of diagnostics were generated by each simulation run. The first was a contour map for each positron disc showing the total amount of charge contained within a given radius as a function of $r$ and $z$. Since the total 
charge of the disk is conserved, contraction of contour lines toward the axis indicates that the disk is being pinched, while the opposite means that the disk is expanding. Because the first positron disk sees the unperturbed electron beam, its contour map should show just betatroin oscillations. Subsequent positron disks wilt have a more complicated time evolution because the electron beam will have been affected by the previous positron disks.

The second set of diagnostics descriljes the "luminosity" $\mathcal{L}$ of each simulation run. I may be defined as the total number of events divided by the cross-section:

$$
\mathcal{L} \equiv \iiint \delta \pi_{+}\{z \cdot t) n_{-}\left(z^{\prime} \cdot t\right) \delta\left(z-z^{\prime}\right\rangle d A d z d z^{\prime}\left|\vec{v}_{+}-\vec{v}_{-}\right| d t
$$

For a flat density profite, $\mathcal{L}=N_{+} N_{-} / A$ where $A$ is the cross-sectional area and $N_{+}$are the total number of electrons and positrons. A contour map of differential Juminnsity as a function of $r$ and positron disc number together with a graph of the luminosity per positron disc constitute the luminosity diagnostics.

The basic results may be sumarized as follows. For initial emittance values much smaller than those corresponding to equilibriuti. the heads of both beams focus down. If $\ell$ is significantly greater $\lambda_{B} / 4$, the beam heads will expand after the initial contraction and the interior portions of the ireams will see a rapidly varying pinch force. This causes a growth in beam emittance which tends to reduce beam luminosity (see Fig. 2). The analogous phenomenon which occurs in stellar systems is called "violent relaxation", and involves a rapidly varying gravitational potential which can thermalize stellar orbits on timescales much shorter than that corresponding to classirai two body interactions. 
Since the luminosity scales inversely with beam area, one wants to pinch the beams as strongly as possible witisent causing too significant an emittance growth. In figure (3), we plot the "nomalized" luminosity versus total particle number for beams with flat and gaussian density profiles. The normalization factor is the luminosity the beams would have had with zero emittance and no particle motion in the transverse plane (i.e. no focusing or expanding). Parameters were chosen for applicability to SLAC: $\gamma=10^{5}, \sigma_{z}=$ $0.1 \mathrm{~cm}, \sigma_{x}=1.5 \cdot 10^{-4} \mathrm{~cm}$, and an initial emittance corresponding to $\sigma_{x^{\prime}}=\frac{d x}{d z}=\sigma_{y^{\prime}}=2 \cdot 10^{-4}$.

In the limit $\mathbf{N}_{+} \rightarrow U_{3}$, the normalized luminosity approaches 1 or less; $1 . e$. there is no beam focusing. For flat density profiles with $\ell=0.1 \mathrm{~cm}$, as $\mathbf{N}_{+}$ increases, so does the normaliz.d luminosity until a peak value of $n 2.1$ is reached for $\ell=\lambda_{B} / 4$. Beyond this ma.imum, the normalized luminosity slowly decreases because the growth in emittance has overwhelmed the initial pinthing.

A slightly largèr maximum increase in normalized luminosity occurs for beams with gaussian density profiles in $z$ and $r$. ïhis increase from $\approx 2$ to 43.5 occurs because the gaussian "tail" in one beam "slowly" compresses the main part of the otiner beam. This slow compression then maximizes the number of interactions in the central regions of the beams where particle densities are the greatest.

For gaussian profiles, there is no obvious decrease of normatized luminosity as $N_{+}+\infty$. (but neither is there any cbvious increase beyond the aforementioned value of $\sim 3.5$ ) as was evident for flat density profiles. This effect is probably due to the wide variation of betatron frequency in $r$ and $z$ -- i.e. outer portions of the beams go through less than one-quarter of an oscillation and are still contracting at the end of the collision whereas the inner portions of the beams have gone through many oscillations and reached equilibrium in emittance value. Figure 4 shows various examples of this. 
In comclusion, we have confirmed $R$. Hollebeek's finding that focusing in the proposed rollided beam experiment can increase the luminosity by as much as a factor of 3 . Maximum luririosity is achieved for gaussian profile beams in whith $\sigma_{2}$ is greater or equal to approximately one-quarter the average hetatron wavelength. For $\sigma_{z}=0.7 \mathrm{~cm}$ and $\sigma_{x}=1.5$ microns, maximum normalized luminosity will occur for $N_{+} \geq 5 \cdot 10^{11}$ particles.

IV. Kink Mode

A pinched beam is generally subject to some forth of the kink (or hose) mode of instability. In the present case it would appear as a sideways thrashirg of the beams about each other. with a drastic rediction of $\mathcal{E}$ if the amplitude of displacement approaches the order of the beam radius. Because finte pulse length ( $($ ) limits instability growth we find by lintiting the interaction period,

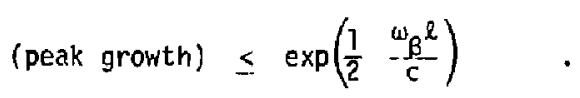

Detaits of cur analys is follow.

As a simple madel let $Y_{+}(z . t)$ be the sideways displacement of the

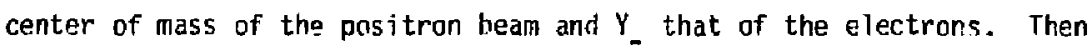
crudely. we have the force law

$$
\begin{aligned}
& \left(\frac{\partial}{\partial c t}+\frac{\partial}{\partial z}\right)^{2} Y_{+}+\alpha\left(\frac{\omega_{B}}{c}\right)\left(\frac{\partial}{\partial c t}+\frac{\partial}{\partial z}\right)\left(Y_{+}-Y_{-}\right)=-\frac{\omega_{B}^{2}}{c^{2}}\left(Y_{+}-Y_{-}\right) . \\
& \left(\frac{\partial}{\partial c t}-\frac{\partial}{\partial z}\right)^{2} Y_{-}+\alpha\left(\frac{\omega_{B}}{c}\right)\left(\frac{\partial}{\partial c t}-\frac{\partial}{\partial z}\right)\left(Y_{-}-Y_{+}\right)=\frac{-\omega_{B}}{c^{2}}\left(Y_{-}-Y_{+}\right) .
\end{aligned}
$$


10

February 8, 1980

The first term on the left of each equation is the convective acceleration, and the term on the right is the restoring force. An additional term. which is first order in the convective derivatives, is included in each equation to model phase mix damping (rate proportion to a $\approx 0.78$ ).

For convenience we define the following variables

$$
\begin{aligned}
& x \equiv \frac{\omega_{B}}{2 c}(c t-z), \\
& y \equiv \frac{\omega_{B}}{2 c}(c t+z),
\end{aligned}
$$

The heads of the beams meet at $c t=0$ and $z=0$ and the tails separate at ct $=\ell, z=0$. The zone of interaction is [see fig. (5)]

$$
\begin{aligned}
& 0 \leq x \leq \frac{\omega_{\beta} l}{2 c} \\
& 0 \leq y \leq \frac{\omega_{\beta} l}{2 c}
\end{aligned} .
$$

Then Ens. (9) and (10) become

$$
\begin{aligned}
& \left(\frac{\partial^{2} Y_{+}}{\partial Y^{2}}\right)_{x}+\alpha \frac{\partial}{\partial y} \quad\left(Y_{+}-Y_{-}\right)=-\left(Y_{+}-Y_{-}\right), \\
& \left(\frac{\partial^{2} Y_{+}}{\partial x^{2}}\right)_{y}+\alpha \frac{\partial}{\partial x}\left(Y_{-}-Y_{+}\right)=-\left(Y_{-}-Y_{+}\right) .
\end{aligned}
$$

We consider a node of the form

$$
\begin{aligned}
& y_{+}=y_{-} \alpha e^{g} \\
& g=-i(\Omega x+\omega y)
\end{aligned}
$$


Eqs. (14) and (15) yield

$$
\begin{aligned}
& \left(1-i \alpha \omega-\omega^{2}\right) Y_{+}-(1-i \alpha \omega) Y_{+}=0 \\
& -(1-i \alpha \Omega) Y_{+}+\left(1-i \alpha \Omega-\Omega^{2}\right) Y_{-}=0
\end{aligned}
$$

The condition for a non-trivial solution is the dispersion relation

$$
\begin{aligned}
0 & =D(\omega, \Omega)=\left(1-i \alpha \omega-\omega^{2}\right)\left(1-i \alpha \Omega-\Omega^{2}\right) \\
& (1-i \alpha \omega)(1-i \alpha \Omega) \\
& =\omega^{2} \Omega^{2}-\omega^{2}-\Omega^{2}+i \alpha\left(\omega^{2} \Omega+\omega \Omega^{2}\right) .
\end{aligned}
$$

The problem now is to find the stationary point of $g$ at given $(x, y)$ subject to the condition that $D=0$; this is equivalent to the usual wave packet or saddle analysis. We use the method of Lagrangian mutipliers:

$$
\begin{aligned}
& 0=\frac{\partial}{\partial \Omega}(g+\lambda D)=-i x+\lambda \frac{\partial D}{\partial \Omega} \\
& 0=\frac{\partial}{\partial \omega}(g+\lambda D)=-i y+\lambda \frac{\partial D}{\partial \omega} .
\end{aligned}
$$

(where $\lambda$ is the undetermined multiplier). Eqs. (21) and (22) yield

$$
\frac{x}{y}=\frac{\partial D / \partial \Omega}{\partial D / d \dot{s}}
$$

For the present study only the case $x=y$ is analyzed. i.e. $z=0$. which is probabiy the ray with maximum grouth. Thus we have from eq. (23)

$$
0=\frac{\partial D}{\partial \Omega}-\frac{\partial D}{\partial \omega}=(\omega-\Omega)[(2 \omega \Omega+2+i \alpha(\omega+\Omega)] \text {. }
$$


which is to be solved along with eq. (20).

The root of eq. (24) $\omega=\Omega$, when plugged into eq. (?3) gives

$$
\Omega^{2}=0 \text {, }
$$

or

$$
\Omega=i \alpha \pm \sqrt{\alpha^{2}+2}
$$

which do not display unstable growth.

For the case $\omega \neq \Omega$, eq. (24) gives

$$
\omega=-\frac{1+\frac{i \alpha}{2} \Omega}{\frac{i \alpha}{2}+\Omega}
$$

Eq. (20) becomes

$$
0=\Omega^{2}-1-\left(\frac{i \alpha}{2}\right)^{2} \Omega^{4}-2\left(\frac{i \alpha}{2}\right) \Omega^{3}-\Omega^{4} .
$$

A useful factorization of eq. (28) is

$$
\left(\Omega^{2}-1\right)^{2}+\Omega^{2}\left(1+\frac{i \alpha}{2} \Omega\right)^{2}=0,
$$

or

$$
\left(\Omega^{2}-1\right) \pm \quad i \Omega\left(1+\frac{i \alpha}{2} \Omega\right)=0 .
$$

The quantity required to compute growth is

$$
X \equiv \Omega+\omega=\frac{\omega^{2}-1}{\frac{i \alpha}{2}+\Omega} .
$$

Eq. (30) may be written

$$
x\left(\frac{j \alpha}{2}+\Omega\right) \pm i\left[\left(\frac{i \alpha}{2}+\Omega\right)+\frac{i \alpha}{2} \times\left(\frac{i \alpha}{2}+\Omega\right)\right]=0,
$$


or

$$
\left[x\left(1 \pm i\left(\frac{i \alpha}{2}\right)\right) \pm i\right]\left[\frac{i \alpha}{2}+\Omega\right]=0 .
$$

Since $\Omega=-\frac{j \alpha}{2}$ is not a possible root we have

$$
x=\frac{\mp i}{1 \pm i\left(\frac{i a}{2}\right)}
$$

Net grouth is then $( \pm+-)$

$$
\begin{aligned}
g & =-i \times x=(-i)\left(\frac{\mp i}{i \pm i\left(\frac{i \alpha}{2}\right)}\right) \frac{\omega_{B}}{2 c} c t \\
& =\frac{1}{1+\frac{\alpha}{2}} \frac{\omega_{B}}{2 c} c t \leq \frac{1}{1+\frac{\alpha}{2}} \frac{\omega_{B}}{2 c} .
\end{aligned}
$$

For the optinal normalized Iuminosity rase of $\ell=\left(\right.$ ? $\left.\pi c / 4 \omega_{\beta}\right)=\lambda_{\beta} / 4$. we have

$$
g \leq \frac{1}{1+\frac{\alpha}{2}} \quad \cdot \frac{\pi}{2}
$$

Thus, a misalignment of the electron and positron beams on the order of $10 \%$ or less should not cause a drastic decrease in luminosity due to the kink instability so long as $\ell \leqslant \lambda_{B} / 4$. 
Figure Captions

Fig. 1 Standard leap-frog algorithm used to advance particle positions and velocities. " $E^{n_{1}}$ refers to the total pinch force, both electric and magnetic, at time step $n$.

Fig. 2 Radial "density" profiles for the first and last positron disc. The contours illustrate the total amount of charge contained within a given radius. The abscissa represents the disc's $Z$ (and thus time) coordinate while the ordinate represents the radial grid number. Note that radial grid $\#$ is equivalent to $r=0$. The initial density profile was flat for both beams, $N_{+}=2.5 \cdot 10^{10}, \ell=0.1$ $\mathrm{cm} \approx \lambda_{B} / 4, r_{0}=1.5 \mathrm{microns}$, and $\sigma_{x}$ corresponds tc $\frac{d x}{d z}=2 \cdot 10^{-4}$. The first positron disc undergoes pure betetron oscillations while the last positron disc has more complicated motion- The difference between the two plots illustrates the focusing effect the position beam has had on the electron beam and the resultant emittance increase in the latter.

Fig. 3 The normalized luminosity $\mathcal{L} / \mathscr{L}_{0}$ is plotted against particle number for flat (solid circles) and gaussian (open circles) density profiles. $\mathscr{L}_{0}$ corresponds to the Tuminosity when the emittance is set to zero and particle orbits are straight lines.

Fig. 4 Same as figure 2 except gaussian density profiles are employed. Here $N_{ \pm}=1.10^{12}, \sigma_{x}=1.5$ microns, $\sigma_{z}=0.1 \mathrm{~cm}$, and 
$\sigma_{x^{4}}=2 \cdot 10^{-4}$. The scale factor for the abscissa is one unit $=0.0166 \mathrm{~cm}$ while that for the ordinate is one un it $=0.276$ microns. The extreme differences between the two plots show the growth in the enittance of the electron bearn and the consequent variation of betatron frequency with $r$.

Fig. $5 \mathrm{~A}(z, c t)$ diagram illustrating the interaction zone and the pseudo-coordinates $x=z-c t$ and $y=z+c t$. 
"Leap-frog" Algarithm

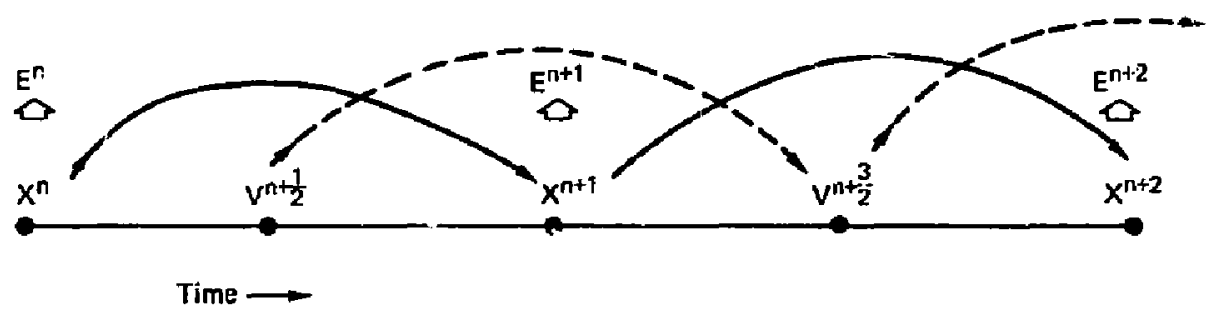

Figure 1 

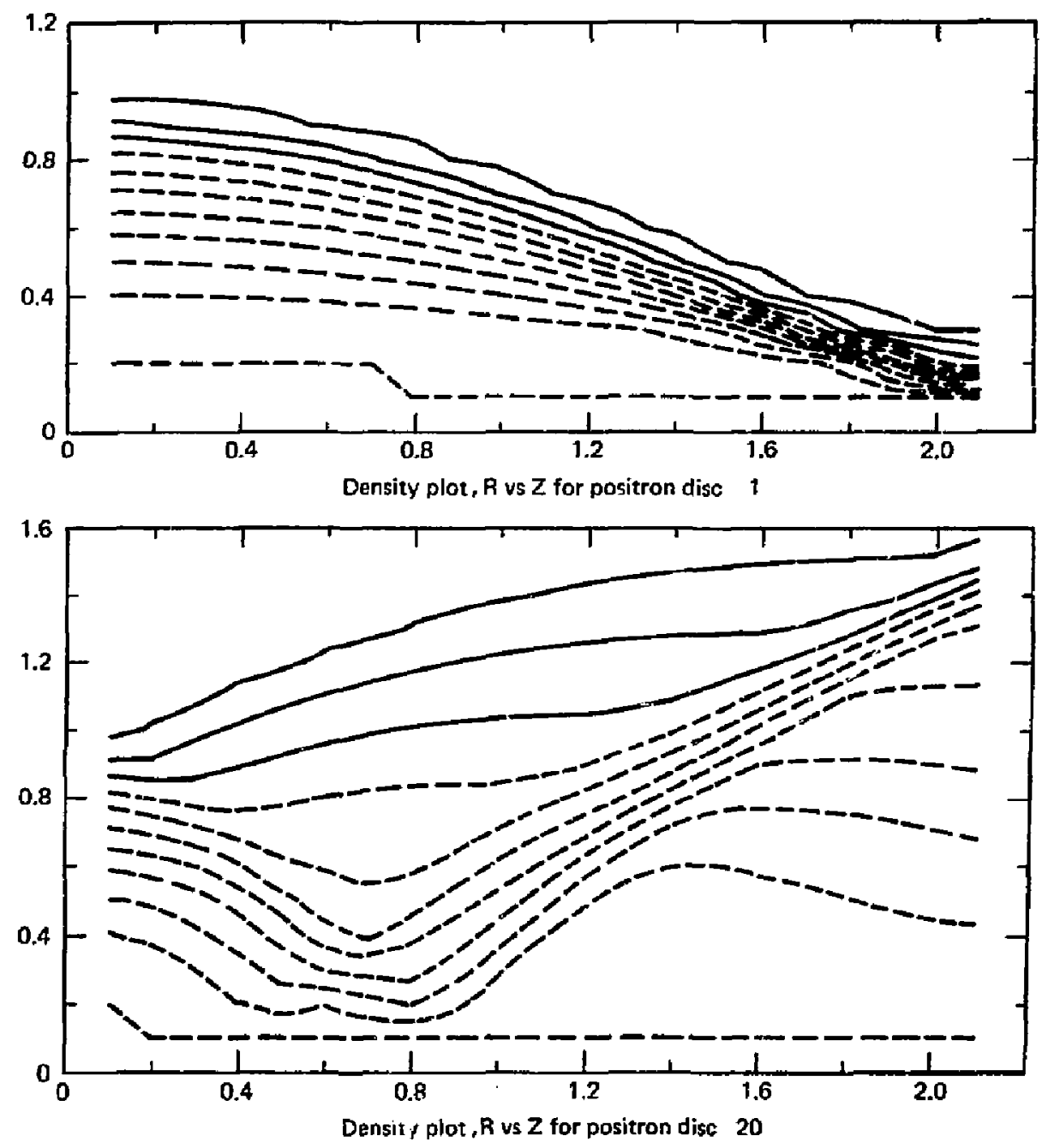

Figure 2 


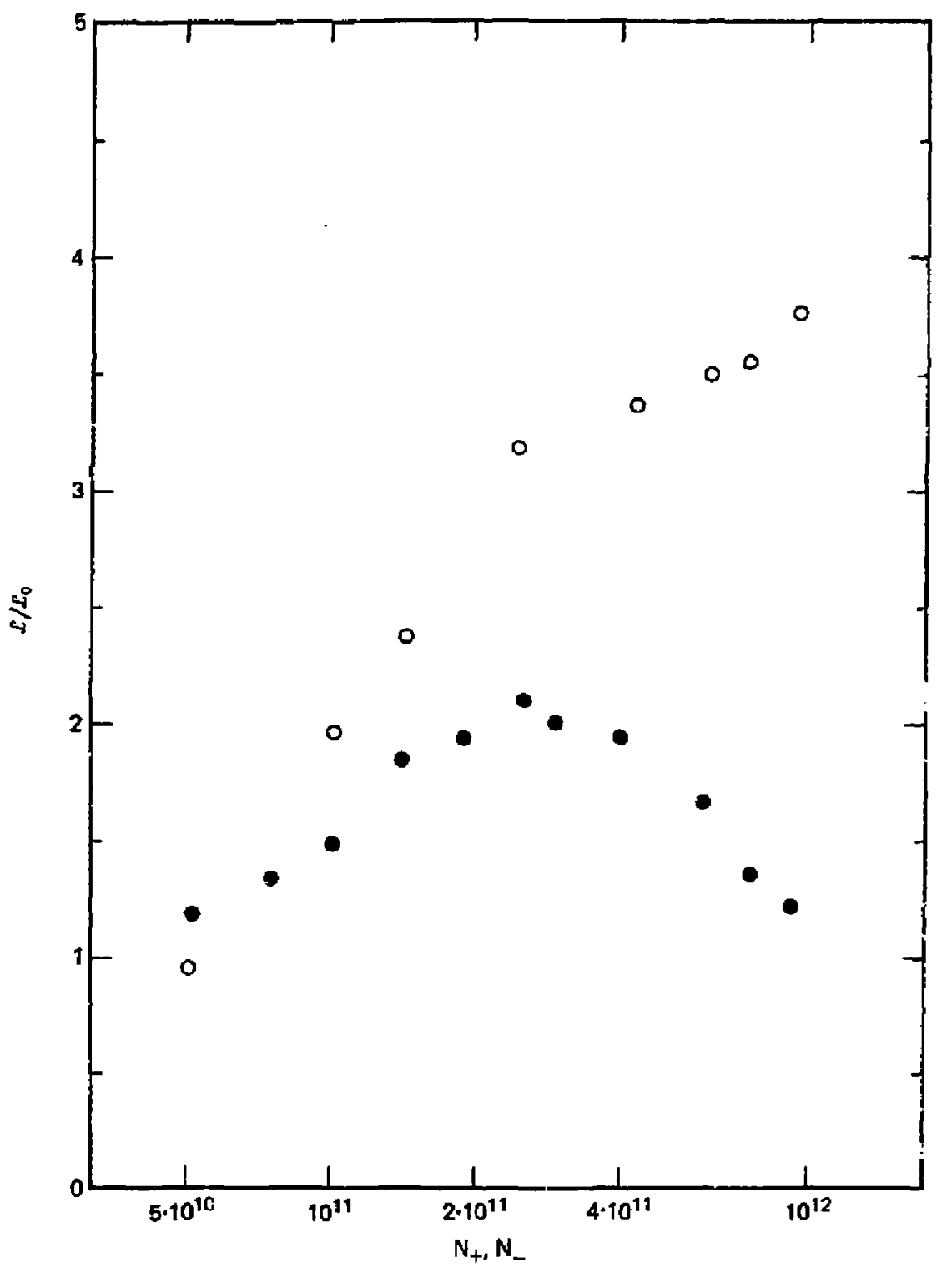

Figure 3 

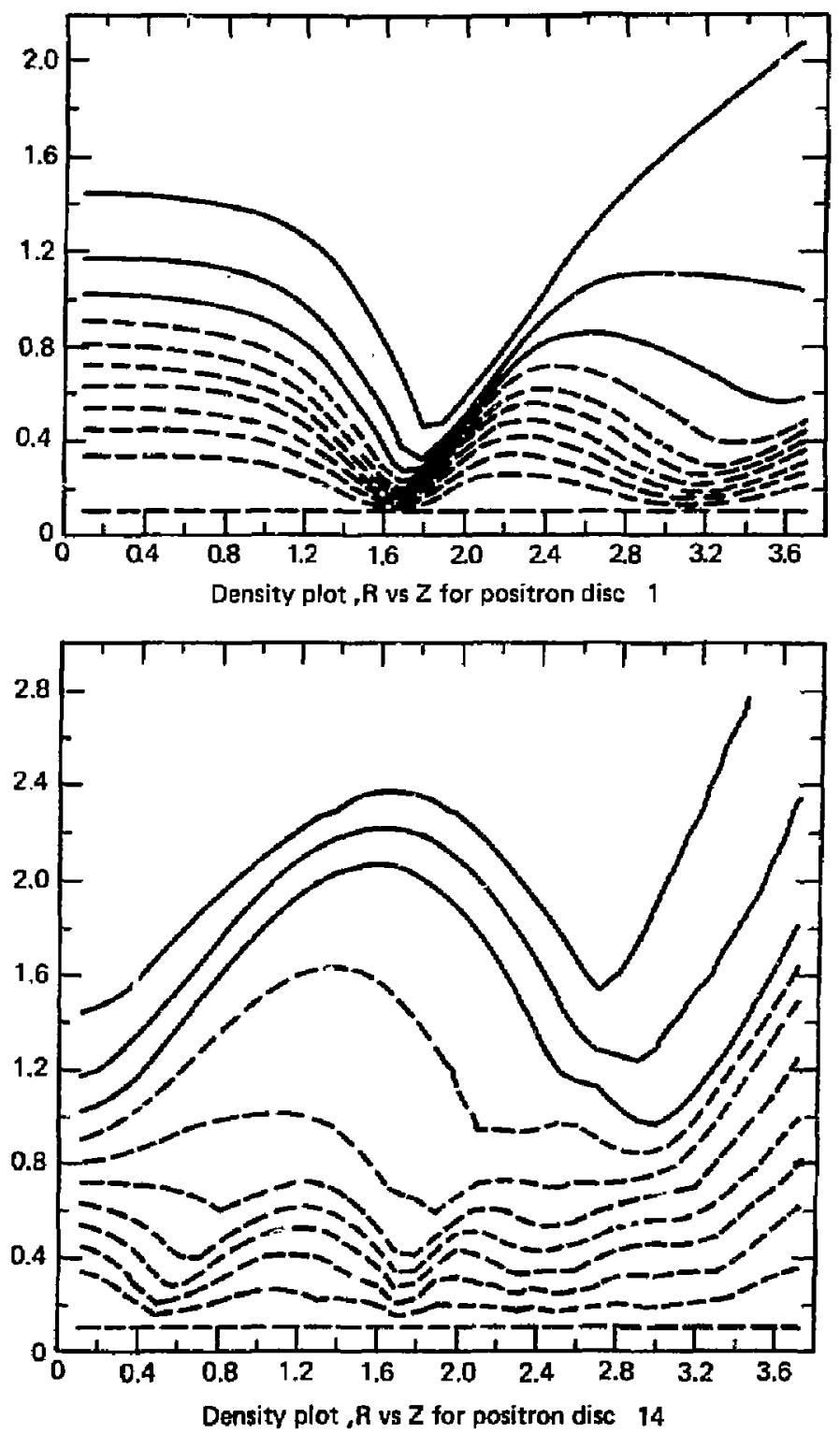

Figure 4 


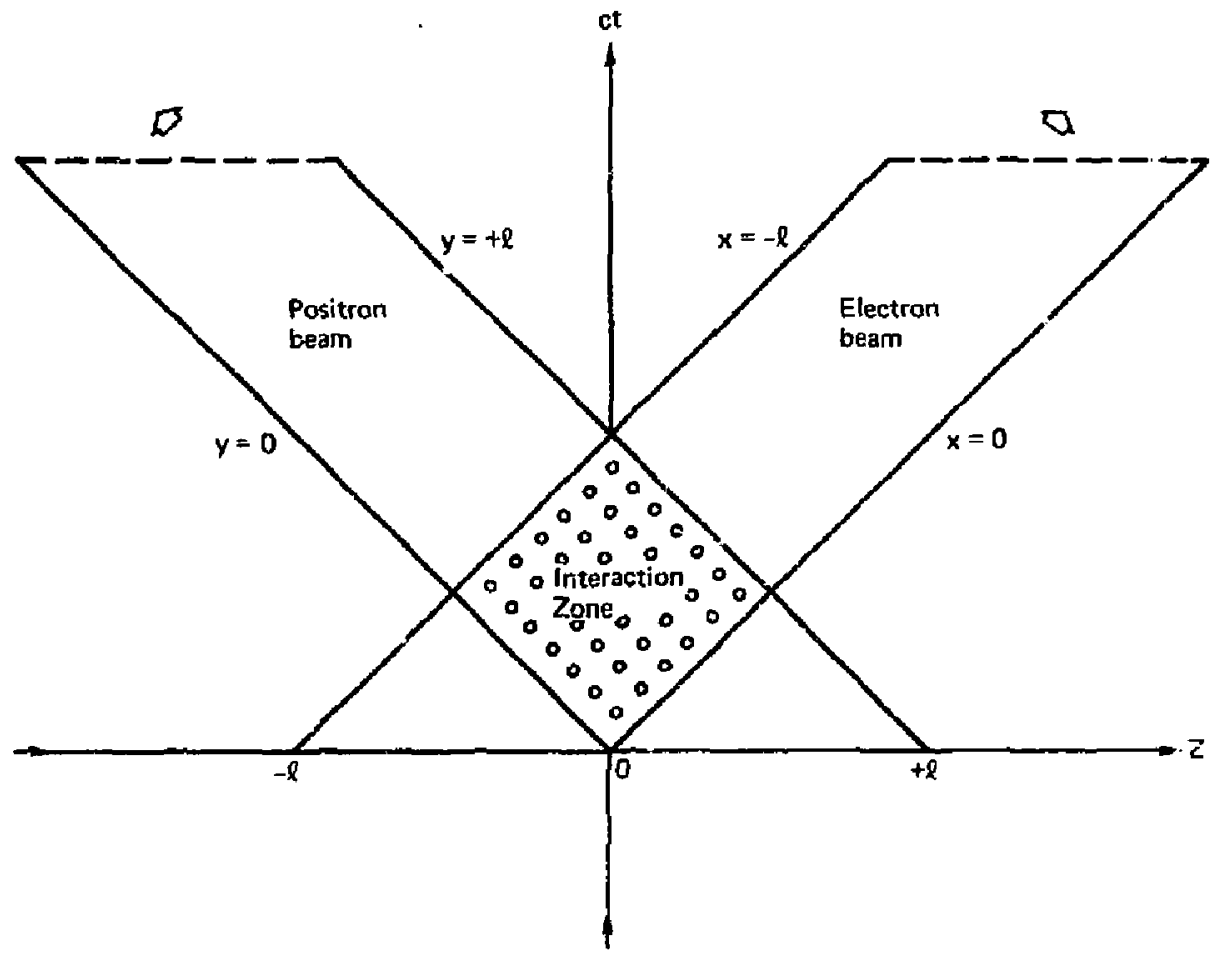

Figure 5 


\section{References}

1. Birdsa11, C.H., and Langdon, A.B., Plasma Physics via Computer Simulation, 1979 edition. These course notes are available from C.K. Birdsall, ités Dept., U.C. Berkeley. 